\title{
Snakes, Ladders, and Information about Sexually Transmitted Infections: Evaluation of a Peer Educator Training on the Thailand-Burma Border
}

\author{
Jillian Gedeon ${ }^{1}$, Jessica Hkum ${ }^{2}$, Saw Nanda Hsue ${ }^{3}$, Meredith Walsh ${ }^{1} \&$ Angel M. Foster ${ }^{1,4}$ \\ ${ }^{1}$ Cambridge Reproductive Health Consultants, Cambridge, MA, USA \\ ${ }^{2}$ Adolescent Reproductive Health Zone, Chiang Mai, Thailand \\ ${ }^{3}$ Independent Consultant, Mae Sot, Thailand \\ ${ }^{4}$ Faculty of Health Sciences, University of Ottawa, ON, Canada \\ Correspondence: Angel M. Foster, DPhil, MD, AM, 1 Stewart Street, Room 312-B, Ottawa, ON K1N 6N5, \\ Canada. E-mail: angel.foster@uottawa.ca
}

Received: February 8, 2016 Accepted: March 23, 2016 Online Published: March 31, 2016

doi:10.5539/gjhs.v8n11p270

URL: http://dx.doi.org/10.5539/gjhs.v8n11p270

\begin{abstract}
The longstanding conflict and civil strife in Burma has had significant consequences on the sexual and reproductive health (SRH) of ethnic minority groups, including adolescents. The Adolescent Reproductive Health Zone in Chiang Mai, Thailand promotes adolescent SRH rights and access to services by having peer educators travel to their hometowns in Burma and lead intensive youth-focused trainings on a variety of topics and issues. In order to evaluate the impact of an intensive three-day workshop dedicated to improving knowledge of sexually transmitted infections (STIs) among peer educators through didactic, experiential, and skill-building exercises, we administered a pre-, post-, and longitudinal assessment. All 13 participants completed both the pre-test and post-test; 11 of 13 participants (85\%) completed the longitudinal evaluation administered three months after the training. Our results indicate that both individual and aggregate STI knowledge increased from baseline and that this knowledge was retained. Moreover, the training increased participants' confidence in their outreach abilities, informed changes in the curricular modules, and led to the implementation of new teaching and learning techniques, especially the incorporation of games and activities. Our findings showcase a successful initiative and suggest similar adolescent peer health educator programs could be undertaken in this protracted crisis and conflict setting.
\end{abstract}

Keywords: health education, myanmar, reproductive health, sexually transmitted diseases, young adults

\section{Introduction}

Burma has suffered from longstanding conflict and civil strife. (Note 1) Forcing ethnic minorities into slave labour, burning crops, and surrounding villages with landmines are but a few of the factors that have resulted in the displacement of millions in both conflict-affected Eastern Burma and neighbouring Thailand (Sietstra, 2012). The political situation has severely compromised the availability of and access to education, food, and healthcare (Back Pack Health Worker Team, 2006). Although recent political reforms in Burma have resulted in greater openness, the lingering effects of pervasive human rights violations and over five decades of conflict are pronounced.

The conflict situation has had significant consequences on the sexual and reproductive health (SRH) of affected populations. Lack of access to comprehensive, medically accurate, and affordable healthcare including family planning services, abortion care, skilled birth attendants, and sexually transmitted infection (STI) prevention and treatment contributes significantly to heightened maternal mortality and morbidity in the Thailand-Burma border region (Back Pack Health Worker Team, 2006; Lee et al., 2006; Mullany et al., 2008). Among the millions who suffer from non-existent or inadequate health services are adolescents who either reside in conflict-affected Eastern Burma (referred to as 'cross border') or in Thailand as refugees or migrants, most of whom are undocumented (Hobstetter et al., 2015; Walsh et al., 2012). Adolescents living along the border are at increased risk of gender based violence and engaging in sex trade and lack of access to information, education, and 
services geared toward youth, both married and unmarried. These dynamics contribute to the unmet need for family planning and SRH care (Benner et al., 2010; Women's Refugee Commission, Save the Children, UNHCR, \& UNFPA, 2012).

In order to address the complex SRH needs of adolescent and young adult populations along the Thailand-Burma border, the Adolescent Reproductive Health Zone (ARHZ) in Chiang Mai, Thailand promotes adolescent SRH rights and access to services and engages in a multitude of efforts to prevent STIs and unintended pregnancy (Hobstetter et al., 2012). Established in 2008, ARHZ represents a grassroots network of six community based organizations serving adolescents aged 12 to 25 (inclusive) in northern Thailand and Eastern Burma (Arnott et al., 2015). ARHZ's reach into rural and remote areas of conflict-affected Burma is facilitated by the longstanding relationships that exist between members and their villages and communities of origin. Peer educators, whose educational backgrounds vary from high school education to university degrees and whose ages can range from 18 to 40, travel annually to their hometowns in Burma and lead intensive youth-focused SRH trainings on a variety of topical areas with the aim of creating a safe and positive space for participants to ask questions and raise issues. This border-based organization reaches more than 1,000 adolescents each year and is one of a small number of organizations that has been able to establish a consistent presence in conflict-affected areas of Eastern Burma (Walsh et al., 2012).

In order to qualify as an ARHZ peer educator, members complete more than 60 hours of initial training related to SRH content areas, counselling, and teaching. This is complemented by ongoing continuing education sessions designed to expose peer educators to additional topical information and provide opportunities for them to hone skills related to teaching and facilitation. Trainings for ARHZ peer educators have been developed over the last decade with input from international experts in adolescent SRH issues and the support of both foreign and local health service professionals and educators. The curriculum used by peer health educators for their sessions in Eastern Burma is developed through collaborative design sessions among network members and then further tailored to meet the specific cultural, linguistic, and language-level needs for different age groups.

In the summer of 2013, a team of reproductive health specialists from Cambridge Reproductive Health Consultants (Cambridge, MA, USA) and the University of Ottawa (ON, Canada) partnered with ARHZ to design and implement a three-day in-service training dedicated to STIs. In this article we present the results of an evaluation of this training and reflect on the aspects of this initiative that were most resonant with peer educators in this protracted conflict setting.

\section{Methods}

\subsection{Description of the STI Training}

ARHZ's leadership team identified the need for an intensive workshop dedicated to STIs after discussion with peer educators and invited a multi-disciplinary team of international collaborators to create, facilitate, and evaluate a training comprised of didactic, experiential, and skill-building components. (Note 2) Our objectives were that participants would: 1) Acquire a more in-depth understanding of STIs, in general, and the five most common STIs in the Thailand-Burma border region, in particular; 2) Learn new ways of communicating information about STIs to adolescents living along the border; and 3) Identify avenues by which access to STI education, prevention, and treatment services could be improved for adolescents in Eastern Burma. The three-day training included evidence-based information on STI prevention, detection, and treatment, discussions of the social, cultural, community, and individual dynamics shaping STIs among adolescents and access to services along the border, and interactive sessions dedicated to active listening, counselling, lecturing, and curriculum design. We employed and modelled a variety of teaching and learning techniques including lectures, facilitated discussions, case studies, games, skits and role playing exercises, individual reflection periods, and question and answer sessions.

\subsection{Training Participants}

Our training targeted existing and experienced ARHZ peer educators and employed a 'train the trainer' model, with the expectation that participants would later incorporate content, educational techniques, and skills into their peer education sessions in Eastern Burma. Thirteen participants attended all components of the three-day training in July 2013 which was primarily conducted in Burmese. In addition to facilitators from the three partnering organizations, the implementation team also included a health educator from Mae Sot, Thailand (SNH) who both translated materials in advance of the training and served as an interpreter during some of the didactic sessions. 


\subsection{Evaluation methods}

In order to evaluate the impact of the training with respect to both knowledge acquisition and implementation of curricular changes, we administered a pre-, post-, and longitudinal assessment. All 13 participants completed both the pre-test and post-test; 11 of 13 participants $(85 \%)$ completed the longitudinal test administered three months after the training. Derived from the materials included in the training, all three versions included 20 true/false, multiple choice, and fill-in-the-blank STI knowledge assessment questions as well as an additional knowledge question related to counselling skills. We also included a section comprised of a five-level Likert item and a series of statements regarding perceptions of STI knowledge and community outreach skills. For the post- and longitudinal tests we expanded this section to include statements about the quality and utility of the training. Finally, in the post- and longitudinal tests we included a set of open-ended questions that focused on the training and the relationship between the training and completed and planned peer education sessions in Eastern Burma (longitudinal only).

We distributed the paper and pencil versions of the test to participants immediately before and immediately after the three-day training; the pre- and post-test took approximately 30 minutes to complete. Three months after the completion of the training we distributed the longitudinal tests to participants; we accepted all tests that were returned to the ARHZ Coordinator within two months of administration (by the end of December 2013). We assigned each participant a unique number at the beginning of the training; we asked participants to include that number on each version of the test so that we could both compare individual results and maintain confidentiality. Training participants were assured throughout the process that the evaluation was voluntary and that they could decline to complete the test and/or decline to answer individual questions.

\subsection{Data Analysis}

Members of our project team translated from Burmese to English all results from the pre-, post-, and longitudinal tests; we then entered our data into a Microsoft Excel® database. In addition to looking at the responses to each individual knowledge assessment question, we collapsed the 20 STI questions to create an overall knowledge score. We calculated means and/or proportions for all three tests at both the individual question and aggregate levels (as applicable) and used paired-t tests to determine significant differences between pre-test/post-test, pre-test/longitudinal test, and post-test/longitudinal test scores. We analysed the responses to open-ended questions included in both the post- and longitudinal tests for both content and themes.

The evaluation initially received approval from ARHZ's leadership for the purpose of informing future programmatic efforts. We subsequently received approval from the Research Ethics Board at the University of Ottawa for the secondary use of the data that was not originally collected for research purposes (File \#H01-14-15).

\section{Results}

\subsection{Knowledge of STIs Improved After the Training}

Thank you for the training. I learned a lot of information. Before I didn't even know what an STD was. (Participant \#1, post-test response)

Results from our assessment demonstrate that compared to baseline, participants' knowledge of STIs improved significantly both immediately and three to five months after the training. As displayed in Table 1, the overall knowledge assessment score for all individual participants increased from baseline to post-test and from baseline to longitudinal test. This was further confirmed by the responses to the open-ended questions in which participants reflected on their perception that the training had led to increased knowledge. However, knowledge level was generally highest among our participants in the immediate wake of the training; of the 11 participants who completed the longitudinal test, six scored highest on the post-test, three scored highest on the longitudinal test, and two revealed no change in knowledge between the post- and longitudinal tests.

These results at the individual level are consistent with the shifts in knowledge in the group as a whole. Out of a possible score of 20, the mean overall pre-, post-, and longitudinal test scores were $11.8(59 \%), 18(90 \%)$, and $16.9(85 \%)$, respectively (Table 1). We found the difference between the pre-test mean knowledge score and both the post-test mean and the longitudinal test mean to be significant $(\mathrm{p}<0.001)$. In contrast, although the mean knowledge score decreased between the post-test and the longitudinal test, this change was not statistically significant. 
Table 1. Overall STI knowledge assessment scores (out of a maximum of 20)

\begin{tabular}{llll}
\hline $\begin{array}{l}\text { Participant } \\
\text { number }\end{array}$ & $\begin{array}{l}\text { Pre-test score } \\
\text { Total (\%) }\end{array}$ & $\begin{array}{l}\text { Post-test score } \\
\text { Total (\%) }\end{array}$ & $\begin{array}{l}\text { Longitudinal test } \\
\text { Total (\%) }\end{array}$ \\
\hline 1 & $7(35 \%)$ & $16(80 \%)$ & $14(70 \%)$ \\
2 & $6(30 \%)$ & $16(80 \%)$ & $\mathrm{n} / \mathrm{a}$ \\
3 & $11(55 \%)$ & $20(100 \%)$ & $15(75 \%)$ \\
4 & $13.5(68 \%)$ & $19(95 \%)$ & $19(95 \%)$ \\
5 & $13(65 \%)$ & $17(85 \%)$ & $20(100 \%)$ \\
6 & $14.5(73 \%)$ & $17.5(88 \%)$ & $20(100 \%)$ \\
7 & $14(70 \%)$ & $17(85 \%)$ & $16(80 \%)$ \\
8 & $13(65 \%)$ & $18(90 \%)$ & $15(75 \%)$ \\
9 & $11(55 \%)$ & $18(90 \%)$ & $\mathrm{n} / \mathrm{a}$ \\
10 & $8(40 \%)$ & $18(90 \%)$ & $11.5(57 \%)$ \\
11 & $14(70 \%)$ & $19(95 \%)$ & $19(95 \%)$ \\
12 & $17(85 \%)$ & $19(95 \%)$ & $19(95 \%)$ \\
13 & $11(55 \%)$ & $20(100 \%)$ & $17(85 \%)$ \\
Mean (\%) & $\mathbf{1 1 . 8 ( 5 9 \% )}$ & $\mathbf{1 8 ~ ( 9 0 \% ) *}$ & $\mathbf{1 6 . 9 ( 8 5 \% ) *}$ \\
\hline
\end{tabular}

* Difference in mean knowledge assessment score when compared to pre-test mean score is statistically significant $(\mathrm{p}<0.001)$.

\section{Participants expressed greater confidence in their knowledge and skills after the training}

The training helps us to learn about more health issues; we can share this information and knowledge [about STDs] with the communities that we work with. (Participant \#3, longitudinal test response)

Consistent with the pre-test knowledge assessment scores, 12 of our participants initially assessed their own knowledge of STIs as fair $(n=9)$ or poor $(n=3)$. Further, 10 of our participants initially rated their ability to conduct community outreach on STI prevention as fair $(n=6)$ or poor $(n=4)$. In the immediate aftermath of the training, all participants reported increased confidence in both their knowledge and outreach abilities by at least one "level" compared to baseline. Reported confidence increased further in the longitudinal assessment; 10 of 11 respondents reported their level of STI knowledge as very good $(n=6)$ or good $(n=4)$ and nine rated their outreach ability as very good $(n=4)$ or $\operatorname{good}(n=5)$.

Our evaluation also reveals that the training helped participants gain knowledge and confidence regarding their listening, counselling, and presentation skills and that they brought those skills to bear on the activities of their organizations. Indeed, of all knowledge assessment questions in the evaluation, the question dedicated to active listening demonstrated the most significant and sustained gain from baseline. Further, as one participant noted in the longitudinal test, "The training was helpful not only for me but also for my organization's work [to] prepare and [to] conduct our own reproductive health training. I also shared the issues with my organization and my community."

\section{Participants incorporated the training into their activities in Eastern Burma}

Snakes [and ladders] game and also drama. And I shared other knowledge from the training in our trainings too. (Participant \#6, longitudinal test response)

Our longitudinal assessment revealed that all 11 respondents had either already incorporated material from the training into their peer health education programs or had plans to do so in early 2014. As one participant noted, "In December [2013], we gave adolescents a reproductive health training in our community [in Eastern Burma]...and we shared all the knowledge that we learned from this training in our training." Participants repeatedly explained that the content dedicated to STIs was particularly useful, citing both specific infections and evidence-based information regarding prevention, as especially relevant for their educational activities. 
However, participants also repeatedly referenced incorporating games and other innovative teaching and learning tools introduced at the training into their programmatic efforts. Of particular note was an STI version of "Snakes and Ladders," depicted in Figure 1. We unveiled this version of the board game, which has long been a childhood favourite in North America and Western Europe, during the training. Some of our participants were unfamiliar with this game at that time but quickly learned the rules and appreciated the way in which the game could be used as a method of interactive knowledge consolidation.

Finally, participants repeatedly explained in their longitudinal test responses that they passed on the information presented in the training to others within their organizations. As one participant explained, "The training is very useful for our organization. I shared the knowledge from the training with my coworkers." Thus some of those who were not able to attend the training had the information shared with them by their colleagues, further expanding the reach of this intensive workshop.

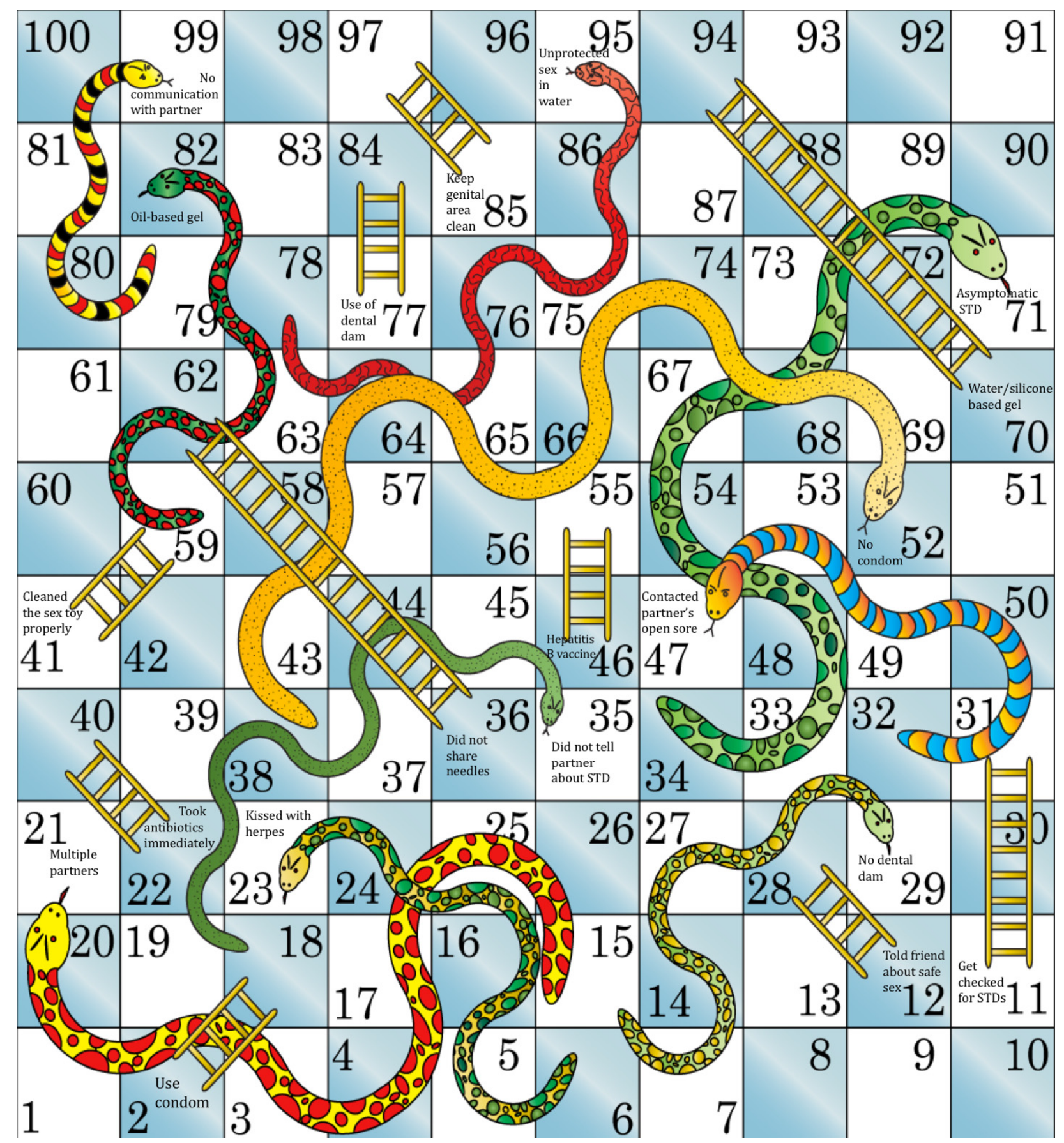

Figure 1. Snakes and ladders, the STI version (in English) 


\section{Discussion}

Globally, adolescents are the age cohort at highest risk for contracting STIs (Hill \& Biro, 2001), with one in twenty contracting a curable infection each year (Dehne \& Riedner, 2005; World Health Organization, 2001). Due to cultural influences and economic dependence, adolescents face unique challenges in gaining access to SRH education (Bott, Jejeebhoy, Shah, \& Puri, 2003). Adolescents living in low-resource and conflict settings face significant barriers to the treatment of STIs due to lack of trained personnel, stigmatization, lower education levels, reduced family and social structures, and lack of access to adequate health care services (Women's Refugee Commission et al., 2012; World Health Organization, 2007).

The use of peer health education programs among youth has been shown to have positive impacts on attitudes and health behaviours, in general, (Sloane \& Zimmer, 1993), and sexual health behaviours, in particular (Kirby, Laris, \& Rolleri, 2007). Peer health education programs in conflict and crisis settings have long been a mainstay of health-related programming, including programs dedicated to hygiene, tobacco use, and SRH issues, although the evidence of impact on behaviour change and community empowerment has been mixed (Ehiri et al., 2014; Drummond et al., 2011; Tanaka et al., 2008). However, the importance of ensuring that peer health educators are qualified, trained, and dedicated has been identified as important factors in influencing adolescent target populations (Women's Refugee Commission et al., 2012).

The results of our assessment suggest that the three-day training improved participants' knowledge of STIs. Not surprisingly, participants' knowledge of the covered subject matter improved immediately after the training when compared to baseline. However, the results show that the mix of audio, visual, and tactile teaching methods facilitated the retention of acquired knowledge three to five months after the training. Further, our assessment reveals that participants integrated the teaching and learning modalities, as well as the content, into their peer health education activities and in-service trainings within their own organizations. Availability of essential SRH services and information currently remains a challenge in this post-conflict region due to structural, service, logistic, and delivery barriers (Hobstetter et al., 2012). That our "train-the-trainer" workshop with 13 participants ultimately reached over 400 adolescents and community health workers in Burmese, Lahu, Shan, Kachin, Karenni, and Thai languages on both sides of the Thailand-Burma border suggests that this type of effort has the potential to meet an identified need.

The feedback on the longitudinal test points to the specific ways in which adolescents benefitted from the community outreach provided by ARHZ members. Members were encouraged to use the presentations and games from the training and replicate them in local languages. Participants in the training adopted games and activities that were most culturally resonant, including Snakes and Ladders and the trivia games. ARHZ members use of variety of methods to teach adolescents about STIs is consistent with the literature showing the importance of using a range of teaching tools and techniques when teaching about SRH issues. Research from a multitude of contexts has shown that sexual health trainings geared towards adolescents that include many forms of teaching, such as group exercises, games, and presentations impacts knowledge acquisition and retention (Gharat, Vasava, Patel, Mihir, \& Modi, 2011; Pearce et al., 2012). Further, research and program evaluations have repeatedly demonstrated the importance of using creative teaching methods that are dynamic and interactive when working with adolescents (Cáceres, Rosasco, Mandel, \& Hearst, 1994; Haignere et al., 2000; Madeni, Horiuchi, \& Iida, 2011; Parwej, Kumar, Walia, \& Aggarwal, 2005). Our findings are consistent with this larger body of literature and indicate that these same dynamics shape the success of initiatives in complex protracted conflict and refugee settings.

\section{Limitations}

Although we believe that the lessons learned from this initiative have import beyond the immediate bounds of the project, our evaluation focused on only one train-the-trainer initiative in Chiang Mai and thus our results cannot be generalized to other organizations working with adolescents in the region. Further, our longitudinal assessment took place three to five months after the training; an additional assessment conducted a year or more after the training would likely shed additional light on both the knowledge and activities of participants in the training. Finally, we conducted both the training and evaluation in Burmese with the assistance of an interpreter/translator. It is certainly possible that content and nuance were lost in translation. However, in working closely with a highly trained interpreter throughout the project and holding a series of team meetings before, during, and after the training, we believe we were able to minimize the impact of this challenge.

\section{Conclusion}

The longstanding conflict in Burma has had a significant impact on SRH among migrants, refugees, and cross-border populations. SRH information and services targeted toward adolescents are minimal in this region, 
but established organizations that reach out to communities to teach medically accurate information, such as ARHZ, can help mitigate the negative consequences of low healthcare resources. The three-day STI training had a positive impact on trainers' knowledge and understanding of STIs and resulted in increased confidence in participants' outreach abilities. SRH trainings that include a combination of audio, visual, and tactile teaching tools appear to be culturally resonant in this setting and effective for both educators and adolescents. Expanding similar efforts in this protracted conflict setting appears warranted.

\section{Acknowledgements}

We received funding for this training from an anonymous donor. Ms. Gedeon's participation in this project was supported by a number of scholarships and grants, including an Ontario Women's Health Scholars Award and a travel scholarship from the University of Ottawa. Dr. Foster's Endowed Chair is funded by the Ministry of Health and Long-Term Care in Ontario and we appreciate the general support for her time that made this project possible. We would like to thank the members of ARHZ for participating in this study as well as Naw Hser Gay Moo for her translation assistance.

\section{Conflict of Interest}

The authors declare that there is no conflict of interests regarding the publication of this paper.

\section{References}

Arnott, G., La, R., Tho, E., Sietstra C., Walsh, M., \& Foster, A. (2015) Establishing a safe abortion referral system for women in Burma residing in Chiang, Mai Thailand: Results of situation analysis research. Cambridge, MA: CRHC.

Back Pack Health Worker Team. (2006). Chronic emergency: Health and human rights in Eastern Burma.

Benner, M. T., Townsend, J., Kaloi, W., Htwe, K., Naranichakul, N., Hunnangkul, S., ... Sondorp, E. (2010). Reproductive health and quality of life of young Burmese refugees in Thailand. Conflict and health, 4(1), 5. http://dx.doi.org/10.1186/1752-1505-4-5

Bott, S., Jejeebhoy, S., Shah, I., \& Puri, C. (Eds.). (2003). Towards adulthood: Exploring the sexual and reproductive health of adolescents in South Asia. Geneva: World Health Organization.

Cáceres, C. F., Rosasco, A. M., Mandel, J. S., \& Hearst, N. (1994). Evaluating a school-based intervention for STD/AIDS prevention in Peru. Journal of Adolescent Health, 15(7), 582-591. http://dx.doi.org/10.1016/1054-139X(94)90143-Q

Dehne, K. L., \& Riedner, G. (2005). Sexually transmitted infections among adolescents The need for adequate health services. Geneva: WHO.

Drummond, P.D., Mizan, A., Brocx, K., \& Wright, B. (2011) Using peer education to increase sexual health knowledge among West African refugees in Western Australia. Health Care for Women International, 32(3), 190-205. http://dx.doi.org/10.1080/07399332.2010.529215

Ehiri, J. E., Gunn, J. K., Center, K. E., Li, Y., Rouhani, M., \& Ezeanolue, E. E. (2014). Training and deployment of lay refugee/internally displaced persons to provide basic health services in camps: A systematic review. Global Health Action, 1(7). http://dx.doi.org/10.3402/gha.v7.23902

Gharat, V., Vasava, B., Patel, S., Mihir, R., \& Modi, B. (2011). Evaluation of pre-test and post-test knowledge questionnaire after intensive ICTC team training among health care workers. National Journal of Community Medicine, 2(1), 161-162.

Haignere, C. S., Gold, R., Maskovsky, J., Ambrosini, J., Rogers, C. L., \& Gollub, E. (2000). High-risk adolescents and female condoms: Knowledge, attitudes, and use patterns. Journal of Adolescent Health, 26(6), 392-398. http://dx.doi.org/10.1016/S1054-139X(99)00090-7

Hill, Y. L., \& Biro, F. M. (2001). Adolescents and sexually transmitted infections. CME Feature.

Hobstetter, M., Walsh, M., Leigh, J., Lee, C., Sietstra, C., \& Foster, A. (2015). Separated by borders, united in need: Emergency contraception access on the Thailand-Burma Border. International Journal of Obstetrics and Gynaecology, 130(Suppl 3), E37-41. http://dx.doi.org/10.1016/j.ijgo.2015.05.008

Hobstetter, M., Walsh, M., Leigh, J., Lee, C. I., Sietstra, C., \& Foster, A. M. (2012). Separated by borders, united in need: An assessment of reproductive health on the Thailand-Burma border.

Kirby, D. B., Laris, B. A., \& Rolleri, L. A. (2007). Sex and HIV Education Programs: Their impact on sexual behaviors of young people throughout the world. Journal of Adolescent Health, 40(3), 206-217. 
http://dx.doi.org/10.1016/j.jadohealth.2006.11.143

Lee, T. J., Mullany, L. C., Richards, A. K., Kuiper, H. K., Maung, C., \& Beyrer, C. (2006). Mortality rates in conflict zones in Karen, Karenni, and Mon states in eastern Burma. Tropical Medicine \& International Health, 11(7), 1119-1127. http://dx.doi.org/10.1111/j.1365-3156.2006.01651.x

Madeni, F., Horiuchi, S., \& Iida, M. (2011). Evaluation of a reproductive health awareness program for adolescence in urban Tanzania-A quasi-experimental pre-test post-test research. Reproductive Health, 8(1), 21. http://dx.doi.org/10.1186/1742-4755-8-21

Mullany, L. C., Lee, C. I., Yone, L., Paw, P., Oo, E. K. S., Maung, C., Lee, T., \& Beyrer, C. (2008). Access to essential maternal health interventions and human rights violations among vulnerable communities in $\begin{array}{lllll}\text { eastern } & \text { Burma } & \text { (Survey). } & \text { PLoS } & \text { Medicine, }\end{array}$ http://dx.doi.org/10.1371/journal.pmed.0050242

Parwej, S., Kumar, R., Walia, I., \& Aggarwal, A. K. (2005). Reproductive health education intervention trial. The Indian Journal of Pediatrics, 72(4), 287-291. http://dx.doi.org/10.1007/BF02724005

Pearce, J., Mann, M. K., Jones, C., van Buschbach, S., Olff, M., \& Bisson, J. I. (2012). The most effective way of delivering a Train-the-Trainers program: A systematic review. Journal of Continuing Education in the Health Professions, 32(3), 215-226. http://dx.doi.org/10.1002/chp.21148

Sietstra, C. (2012). The Thailand-Burma border: Addressing needs in long-term conflict settings. In A. M. Foster \& L. L. Wynn (Eds.), Emergency Contraception: The story of a global reproductive health technology (pp. 239-253). New York: Palgrave MacMillan. http://dx.doi.org/10.1057/9781137016485.0023

Sloane, B. C., \& Zimmer, C. G. (1993). The Power of Peer Health Education. Journal of American College Health, 41(6), 241-245. http://dx.doi.org/10.1080/07448481.1993.9936334

Tanaka, Y., Kunii, O., Hatano, T., \& Wakai, S. (2008). Knowledge, attitude, and practice of HIV prevention and HIV infection risks among Congolese refugees in Tanzania. Health \& Place, 14(3), 434-452. http://dx.doi.org/10.1016/j.healthplace.2007.07.005

Walsh, M., Kellock, W., Hai, T., Lagone, E., Sietstra, C., Lessard, D., \& Foster, A. M. (2012). Protecting our future: Adolescent reproductive health and rights on the Thailand-Burma border. Thailand: Adolescent Reproductive Health Network.

Women's Refugee Commission, Save the Children, UNHCR, \& UNFPA. (2012). Adolescent sexual and reproductive health programs in humanitarian settings: An in-depth look at family planning services.

World Health Organization. (2001). The second decade: Improving adolescent health and development.

World Health Organization. (2007). Global strategy for the prevention and control of sexually transmitted infections: 2006-2015: Breaking the chain of transmission.

\section{Notes}

Note 1. In 1989, the military junta changed the official name of the country to 'Myanmar.' Although many governments and the United Nations have recognized this change, numerous organizations operating in Thailand continue to use the term 'Burma.' Out of respect for our local partners, we have chosen to use 'Burma' throughout this article.

Note 2. Consistent with international norms, we use the term 'sexually transmitted infection' throughout this article. However, the term 'sexually transmitted disease' and more specifically the abbreviation STD is widely used by health organizations operating along the border. Our training materials and the responses of participants reflect this linguistic reality.

\section{Copyrights}

Copyright for this article is retained by the author(s), with first publication rights granted to the journal.

This is an open-access article distributed under the terms and conditions of the Creative Commons Attribution license (http://creativecommons.org/licenses/by/3.0/). 